\title{
The Karlsruhe Dynamo Experiment
}

\section{U. Müller and R. Stieglitz}

Received: 23 October 2001 - Accepted: 6 December 2001

\begin{abstract}
It has been shown theoretically in the past that homogeneous dynamos may occur in electrically conducting fluids for various vortical velocity fields. Roberts (1972) investigated spatially periodic, infinitely extended fields of vortices which Busse $(1978,1992)$ confined to a finite cylindrical domain. Based on Busse's vortex arrangement a conceptual design for an experimental homogeneous dynamo has been developed and a test facility was setup at the Forschungszentrum Karlsruhe. The first experiments demonstrated that permanent dynamo action can be generated in a cylindrical container filled with liquid sodium in which by means of guide tubes counterrotating and countercurrent spiral vortices are established. The dynamo is self-exciting and the magnetic field saturates at a mean value for fixed supercritical flow rates. The instantaneous magnetic field fluctuates around this mean value by an order of about 5\%. As predicted by theory the mode of the observed magnetic field is non-axisymmetric. In a series of experiments a phase- and a bifurcation diagram has been derived as a function of the spiral and axial flow rates.
\end{abstract}

\section{Introduction}

Today it is generally accepted that planetary magnetic fields originate from vortex-type flow of electrically conducting fluids. From general physical and physico-chemical considerations it has been concluded that in rotating planets vortical flow is generated in a liquid core by thermal and solutal convection (see, e.g. Braginsky and Roberts, 1995). Busse and Carrigan (1976) have demonstrated experimentally that in rapidly rotating spherical shells a convective flow driven by density gradients may form a columnar vortex pattern typically oriented parallel to the rotation axis.

Naturally the question arises, whether a regular spatial arrangement of spiral vortices is capable to amplify small mag-

Correspondence to: R. Stieglitz

(robert.stieglitz@iket.fzk.de) netic seed fields to a finite intensity, depending on the conversion rate of mechanical into electrical energy and the dissipation rate of the dynamo system. Roberts $(1970,1972)$ studied analytically dynamo action associated with an infinitely extended and with regard to two directions spatially periodic velocity field characterized by a velocity scale $u$ and a wavelength $2 a$. Together with the magnetic diffusivity of the fluid $\lambda$ these parameters can be combined in a dimensionless group, the magnetic Reynolds number $R e_{m}=$ $(u \cdot a) / \lambda$, to characterize the system. Roberts shows that "almost all spatially periodic velocity distributions in a homogeneous conducting fluid will generate dynamo action for almost all values of the conductivity". Busse $(1978,1992)$ modified Roberts' dynamo model by introducing a second larger length scale, say the radius $r_{0}$ (see Fig. 1a). He assumes $a / r_{0} \ll 1$, and using a scale separation method he derives approximate conditions for the onset of magnetic self-excitation and the solution for the associated magnetic field pattern. Introducing magnetic Reynolds numbers for the mean axial and the mean azimuthal velocity components $\left(u_{C}, u_{H}\right)$ in the form $R e_{m C}=\left(u_{C} \cdot a\right) / \lambda$ and $R e_{m H}=\left(u_{H} \cdot d\right) / \lambda$ he finds as a condition for the occurence of an axisymmetric field,

$R e_{m C} \cdot R e_{m H} \geq \frac{32}{\pi}\left[1+\left(\frac{3.83}{\pi} \cdot \frac{d}{r_{0}}\right)^{2}\right]$,

where $d$ is the axial length of the cylindrical domain. Based on the two-scale dynamo model Busse (1992) proposed a conceptual design for an experimental validation of this idea. Relationship (1) suffers from several limiting assumptions concerning realistic boundary and matching conditions as well as the mean field approximation itself which were addressed by Busse et al. (1996) and Rädler et al. (1996, 1998). Nevertheless, it has been employed to optimize the container and the channel geometry with regard to the pumping power, as it contains the crucial length scales $d, r_{0}$ and $a$ and the measures for the axial and azimuthal flow rates $V_{\text {Central }}$ and 


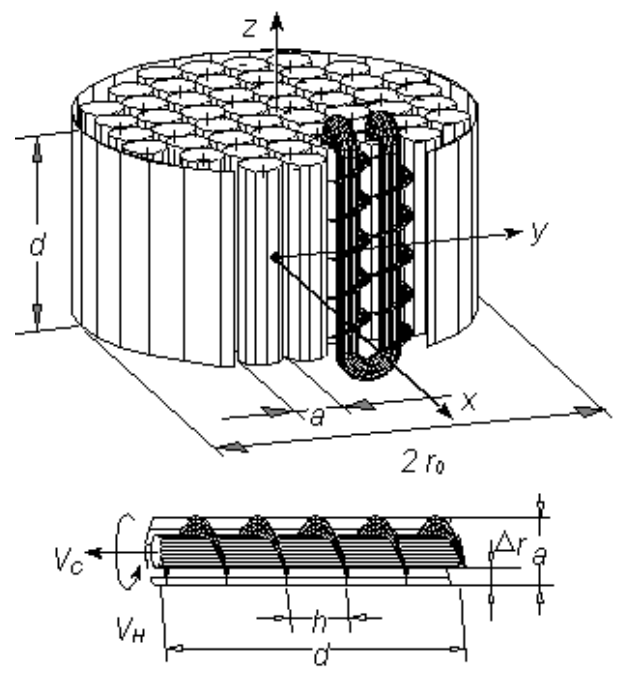

(a)

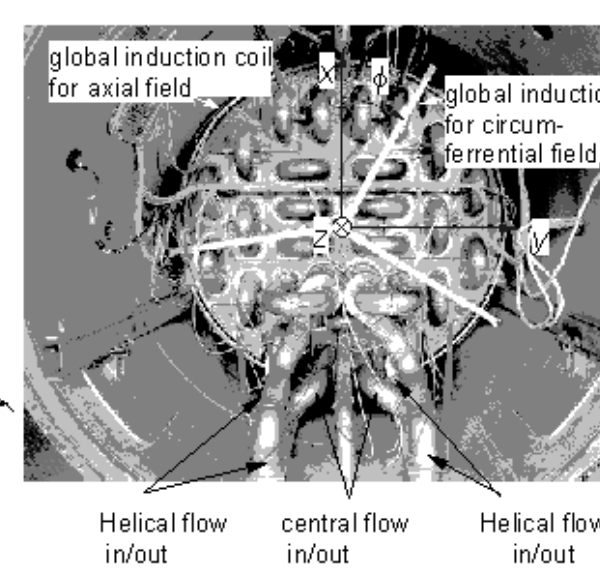

(b)

Fig. 1. Conceptional engineering design for a dynamo test module according to Stieglitz and Müller (1996), (a) vortex generator and vortex generator assembly, (b) Photograph of the operational Karlsruhe Dynamo test module. A reference coordinate system is marked.

$V_{\text {Helical }}$, which are related to the axial and azimuthal velocties $u_{C}$ and $u_{H}$ of the model flow.

In order to assess constraints of Relationship (1) Tilgner (1997) and Rädler et al. (1996, 1998) performed more detailed calculations for technical geometries which account for realistic matching conditions for the magnetic field from the inside to the outside domain of the dynamo module and also for the sodium filled zones at rest within the module as well as for connecting bends and pipes. As a result of their calculations it turned out that the lowest critical magnetic Reynolds numbers $R e_{m C}$ and $R e_{m H}$ are obtained for the lowest non-axisymmetric mode $m=1$ rather than for the axisymmetric mode $m=0$ of the magnetic field. The results of Rädler et al. (1996, 1998) and Tilgner (1997) will be used for comparison with experimental results.

\section{Experimental facility}

The dynamo test module was designed under the viewpoint that for an available pumping capacity a sufficient flow rate for a self-excitation of a magnetic field can be achieved. This required a fluid dynamic optimization of the vortex generators with regard to the pressure losses. A semi-technical sketch of the module and the coordinate system with its origin located in the module's center is shown in Fig. 1. In a test series a pressure drop correlation for the flow in the helical ducts was derived containing the following parameters: $Z$ the number of vortex generators, $\chi$ the number of windings per vortex generator, $r_{m}$ the mean radius of the helical duct, $\Delta r$ the distance between inner and outer tube, $h$ the height of each winding and $\rho$ the density of the liquid sodium. Using Relation (1) and the derived pressure drop correlation the optimization results in critical magnetic Reynolds numbers $R e_{m}$ of less than 10 or in technical terms in volumetric flow rates required for self-excitation of $V_{\text {Central }}=V_{\text {Helical }}=115-143 \mathrm{~m}^{3} / \mathrm{h}$ for the following geometrical dimensions: $Z=52, a=0.21 m, h=0.19 m$, $\Delta r=0.055 m, r_{0}=0.85 m, d=0.703 m$ and $\chi=3.7$. The module is entirely fabricated of stainless steel. The module is placed in a room in which the average magnetic field strength is less than 0,5 Gauss. A power test of the sodium induction pumps showed that the magnetic perturbations produced by them do not affect the locations where the measurements of the dynamo action are conducted. The dynamo's magnetic field is measured by six Hall-probes resolving less than 0.5 Gauss and, moreover, sensed by six global induction coils. The sodium volumetric flow rate for the test module is provided by MHD pumps in three independent loop systems and is measured by electromagnetic (EM) flow meters in each loop which are calibrated to have errors less than $0.3 \%$. The pressure drop across the module in each of the three independent flow channels were measured by using sensitive capacitance pressure gauges with an accuracy of $\pm 5 \cdot 10^{2} \mathrm{~Pa}$. A more detailed description of the experimental set-up and the sodium systems may be taken from a report by Stieglitz and Müller (1996). Dynamo action in this test facility was demonstrated in late 1999 and first experimental results have been published by Stieglitz and Müller (2001). Here we present some results from recent experiments.

\section{Results}

Figures $2 b-d$ show time series of the signals of six Hallprobes located in a tube on the center axis of the module at different positions. The gradual increase of the volumetric flow rates in the central duct $\left(V_{\text {Central }}\right)$ and the helical channels $\left(V_{\text {Helical, } 1}, V_{\text {Helical,2 }}\right)$ is shown in Fig. 2a as a function of time. The graphs in Figs. $2 b$ and $c$ show as a response the 


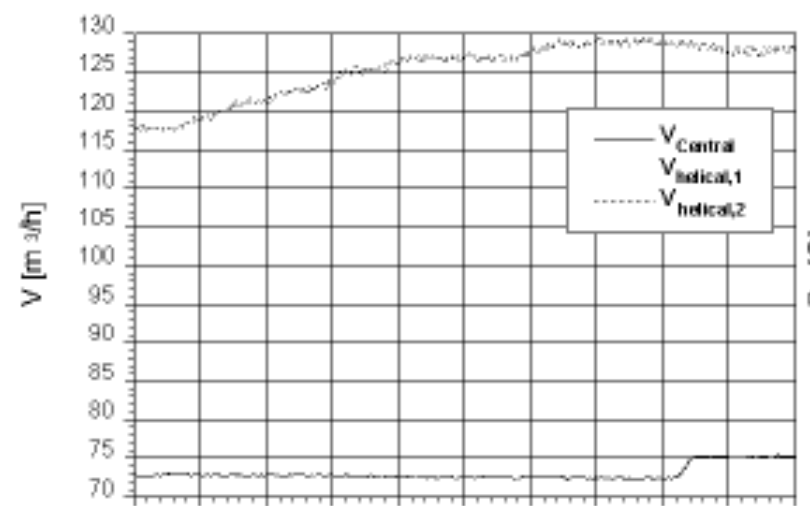

(a)

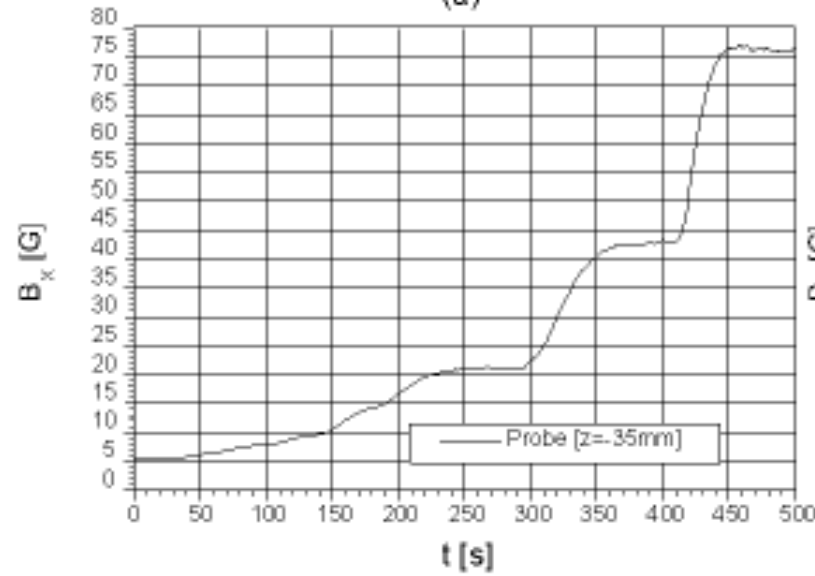

(c)

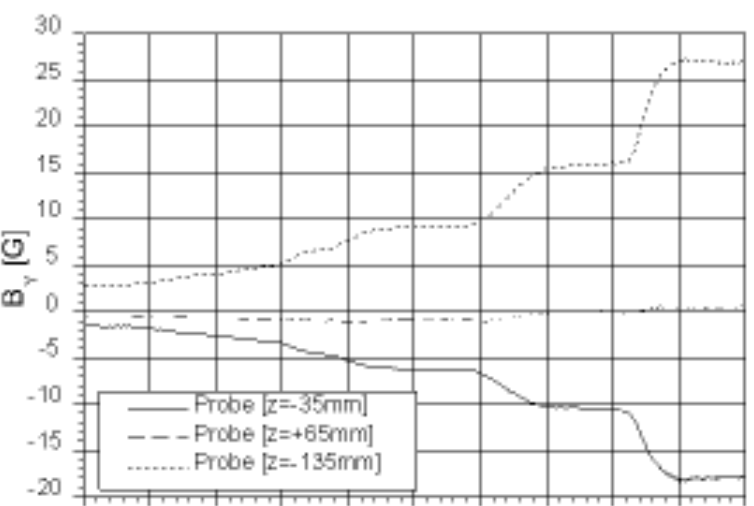

(b)

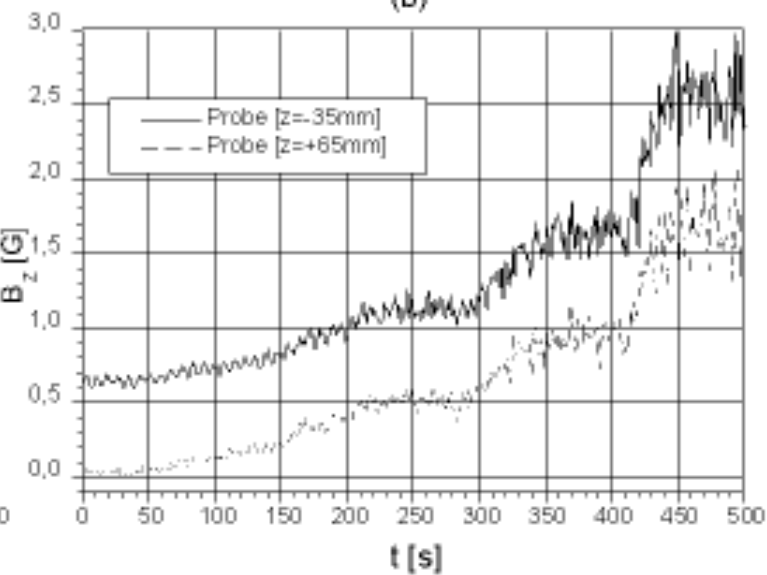

(d)

Fig. 2. (a) Time averaged signals of the volumetric flow rates in the central $\left(V_{\mathrm{Central}}\right)$ and the helical ducts $\left(V_{\mathrm{Helical}, 1}, V_{\mathrm{Helical}, 2}\right)$. (b)-(d) Corresponding signals of three Hall probes $\left(z=-135 \mathrm{~mm}, z=-35 \mathrm{~mm}\right.$ and $z=65 \mathrm{~mm}$ ) indicating the three $B$-field components $B_{x}$ (b), $B_{y}(\mathrm{c})$ and $B_{z}(\mathrm{~d})$ according to the coordinates defined in Fig. $1 \mathrm{~b}$.

step-like increase of two components of the magnetic field in $x$ and $y$ direction. These figures demonstrate the presence of a homogeneous dynamo producing a mean magnetic field of the order of 80 Gauss. Moreover, each increase of the volumetric flow rate to the next level is followed by a corresponding increase of the magnetic field to another saturation level. There is a delayed response of the $B$-field to the controlled variation of the volumetic flow of about 2-3 times the variation interval of the flow. It is to be noted here that no additional external magnetic field was supplied to the test module during this run aside from the ambient seed field of less than 0.5 Gauss.

Two other Hall probes sensitive to the $z$-component of the magnetic field did not record signals of comparable intensity. Figure $2 \mathrm{~d}$ shows the corresponding axial magnetic field intensity in the center bore of the module. The relatively low intensity of the axial field component together with the high intensities of the other field components indicates the non-axisymmetric nature of the generated magnetic field and thus supports the theoretical predictions of Tilgner (1997) and Rädler et al. (1996, 1998). Their calculations show that for the mean field the non-axisymmetric mode $m=1$ is preferred. Another experimental evidence for the presence of the mode $m=1$ is shown by the photograph in Fig. 3b and the isoline graph in Fig. 3c. An array of compass needles (located in the plane $y=1.5 \mathrm{~m}$ ) was photographed at a super-critical state of the dynamo. The photograph indicates a source (sink) of lines of force near the $y$-axis emerging from the center of the module.

The graph in Fig. 3c shows the normal component of the $B$-field on the same plane measured by a "carry on" Gauss meter. The isolines clearly indicate the quasi-dipole axis to be perpendicular to the axis of the module. Moreover, the quasi-dipole axis is slightly shifted towards the fourth quadrant of the plane. Whether this effect reflects small technical non-symmetries of the module or generic non-symmetic properties of the velocity field has to be shown by further analyses.

Hall-probe signals for the saturated magnetic field at fixed super critical flow rates show characteristic fluctuations with different time scales. In Fig. 3a the time series of three simultaneously recorded Hall-probe signals are presented. Preliminary assessments have given that the frequency of the magnetic field fluctuations is much smaller than that of the pressure fluctuations caused by the feeding pumps, which have a typical time period of the order $0.1 \mathrm{~s}$. Moreover, the 


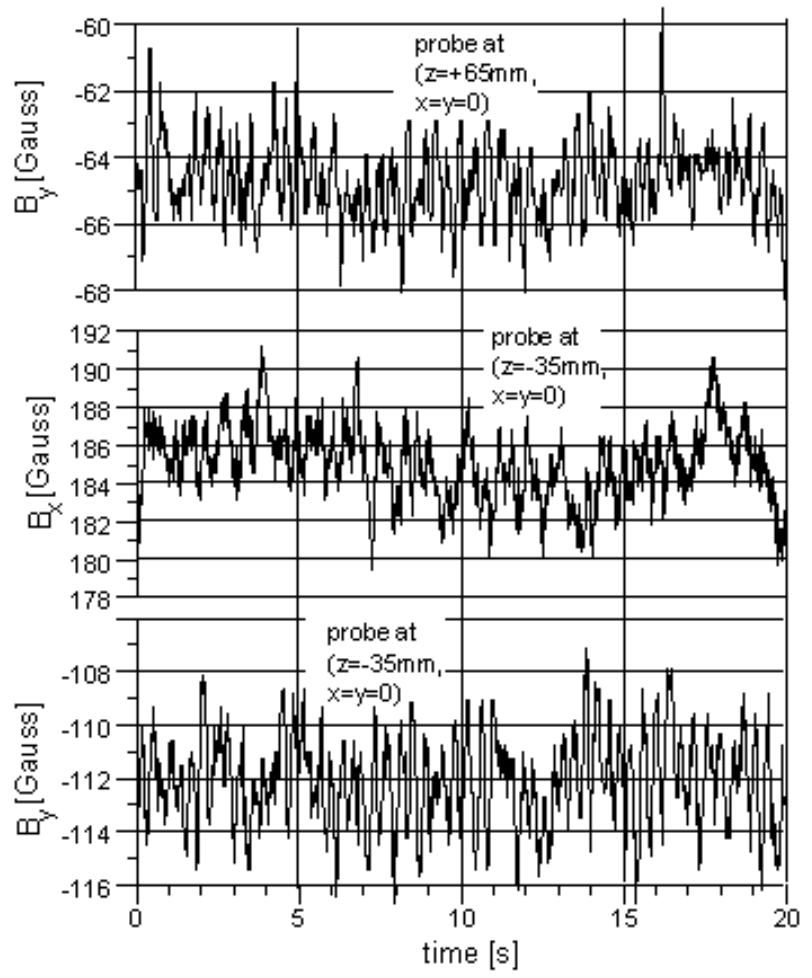

(a)

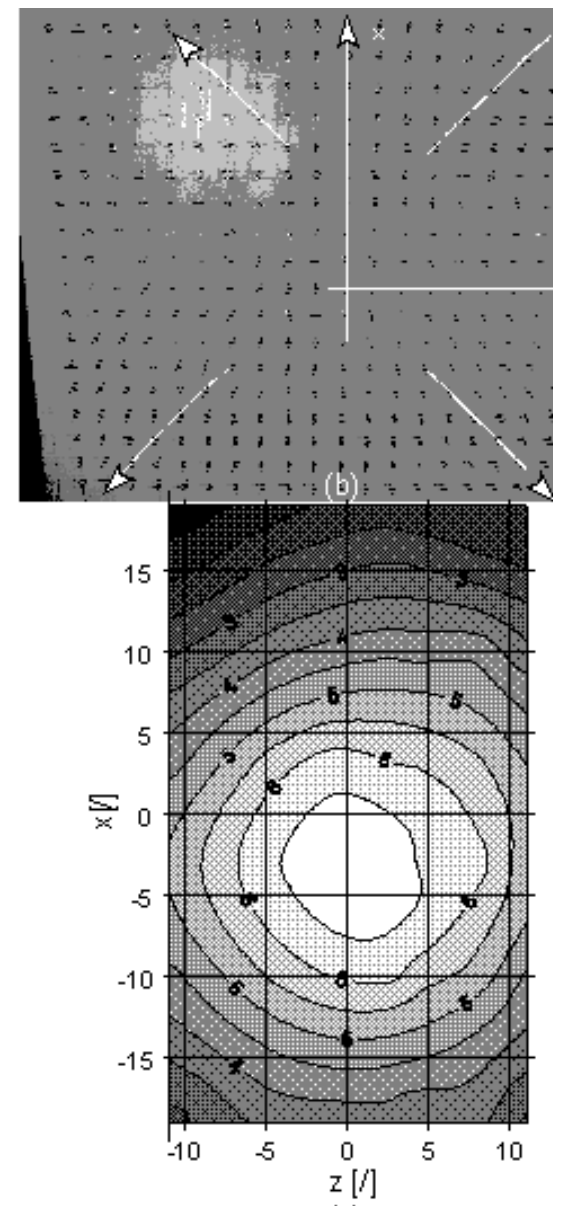

(c)

Fig. 3. (a) Simultaneously recorded time series of different Hall-probes and different magnetic field directions for the volumetric flow rates $V_{\text {Central }}=134 \mathrm{~m}^{3} / \mathrm{h}, V_{\text {Helical }}=101 \mathrm{~m}^{3} / \mathrm{h}$. (b) Photograph of an array of compass needles indicating the magnetic field line structure in the plane $y=1.5 \mathrm{~m}$ at the same conditions. (c) Isolines of the normal component of magnetic field in the same plane.

amplitude of the magnetic field fluctuations is much larger than the resolution limit of the Hall-probes. First time series analyses suggest that the signals of the probes seem to be cross-correlated and characteristic frequencies seem to be related to the transport velocities of the fluid and to the magnetic field within the module. However, more detailed analyses have to be performed in the future.

A characteristic feature of the observed dynamo action is the measured significant increase of the pressure losses in the flow channels of the test module after the onset of selfexitation. The increase is caused by the occurence of Lorentz forces which gives rise to an additional magnetic pressure. These additional MHD-pressure losses can be recognized from the insert graph in Fig. 4 where, as an example for a fixed flow rate in the central loop, i.e. $V_{\text {Central }}=105 \mathrm{~m}^{3} / \mathrm{h}$, the pressure losses accross the module in the helical loops $\Delta p_{\text {Helical }}$ are given as a function of the volumetric flow rate $V_{\text {Helical }}$. The sudden change in the slope of the pressure loss curve indicates the transition from a pure non-magnetic to a magneto-fluiddynamic state. The intersection of the two curves is assumed to define the point of transition. By mea- suring the pressure losses for various helical and fixed central flow rates a phase diagram for dynamo action was derived for our test module. This can be seen from Fig. 4.

The interpolation line between experimentally determined points of transition separates the domains in the $V_{\text {Central }}$ and $V_{\text {Helical }}$ plane for which self-exitation of magnetic fields is possible or not. For comparison, Fig. 4 shows also the curves for onset of dynamo action predicted by the numerical model calculations of Tilgner (2000). The theoretical curve which best fits the experimental data takes into account a correction of $10 \%$ of the molecular value of the magnetic diffusivity of sodium due to turbulence.

At a closer look to an actual point of transition defined by the pressure criterion we observe in reality a smooth transition. This is seen more clearly from the local measurement of the magnetic field at saturation near the center of the module. Figure 5 shows as a typical case the measured $y$-component of the $B$-field as a function of the flow rate $V_{\text {Helical }}$ at a fixed central flow rate $V_{\text {Central }}=105 \mathrm{~m}^{3} / \mathrm{h}$. The curves fitted to the experimental points exhibit characteristic features of a non-perfect transcritical pitchfork bifurcation from a basic 


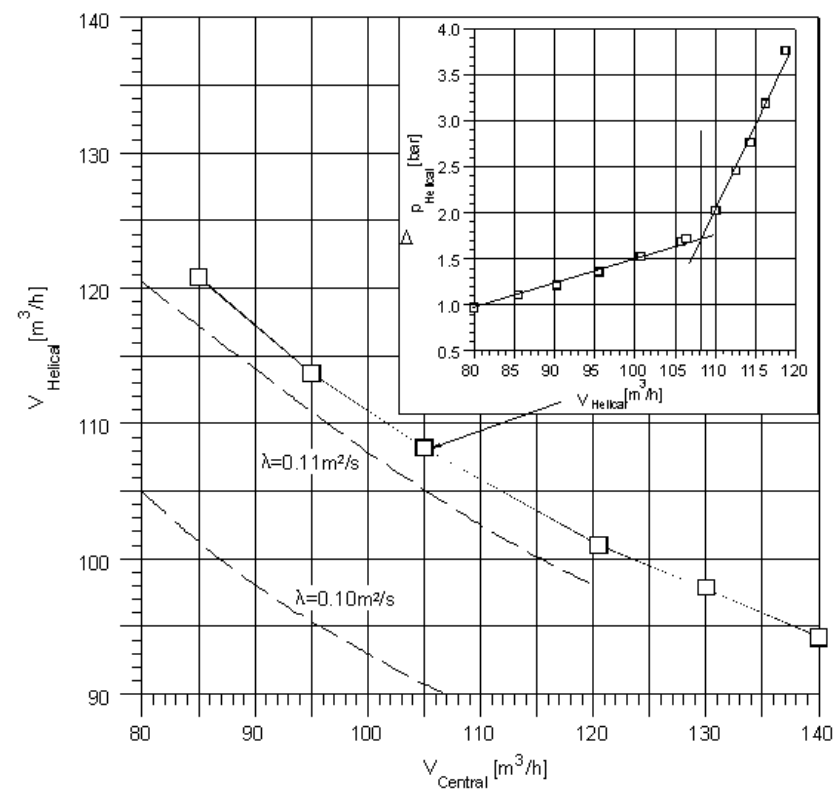

Fig. 4. Phase diagram of dynamo action as a function of the central and helical flow rates $\left(V_{\text {Central }}, V_{\text {Helical }}\right)$. The subgraph illustrates the method to determin the stability limit at $V_{\text {Central }}=105 \mathrm{~m}^{3} / \mathrm{h}$. The dashed lines represent model calculations of Tilgner (2000) for two different values of the magnetic diffusivity $\lambda$.

state, here the pure hydrodynamic state. There are on the one side the continuously emerging dynamo states indicated by the slowly increasing $B$-field for lower helical flow rates and drastically increasing values beyond a certain threshold flow rate. On the other side we find also saturated dynamo states which have a magnetic field of opposite direction. They exist only above a certain value of the flow rate. These states can not be obtained experimentally by increasing the flow rates in a quasi-steady way from subcritical to supercritical conditions. They represent the stable part of the isolated branch of an imperfect bifurcation graph. In our experiment these magnetic states were enforced by imposing suddenly for a short period of time an external magnetic field of opposite direction to a dynamo state on the continuous branch. The external magnetic field was generated by two properly arranged Helmholtz-coils close to the test module. Depending on the intensity of the external $B$-field a transition could be realized from states on the continuous branch to states on the isolated one. Furthermore, we find that the dynamo magnetic field changes direction in a transient, if the helical flow rates are reduced below a certain threshold value, thus indicating the existence of a the turning point in the bifurcation diagram. The observations show that after the transient the previous states on the continuous branch is not exactly recovered, rather a magnetic field of lower intensity is found. This phenomenon has to be investigated in more detail furtheron.

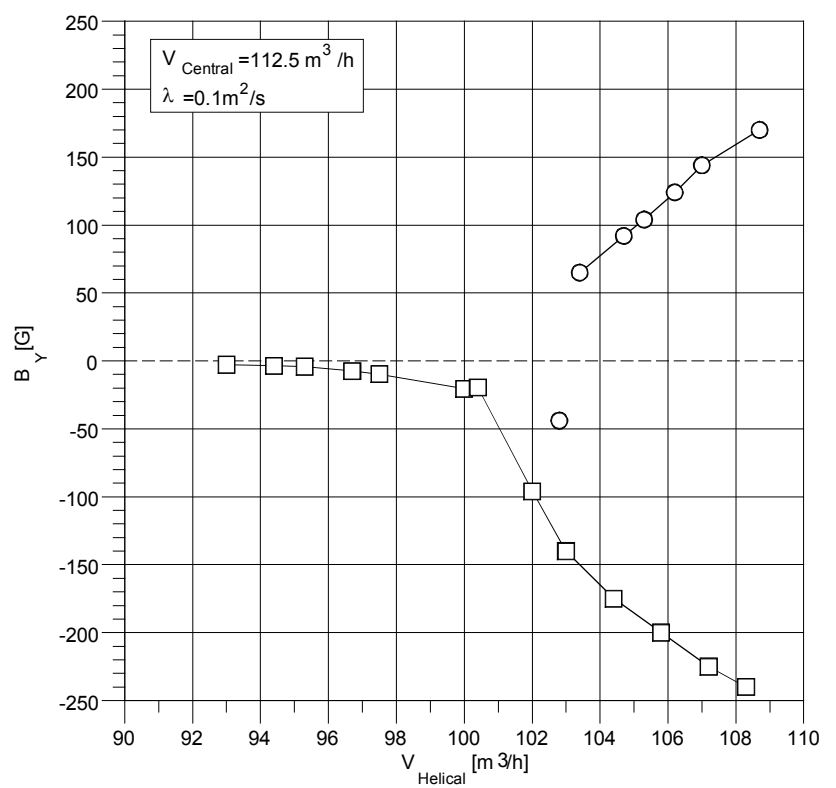

Fig. 5. A typical bifurcation diagram of the Karlsruhe dynamo. Here, the measured magnetic field intensity in $y$-direction $\left(B_{Y}\right)$ at $x=y=z=0$ is shown as a function of the helical flow rate $\left(V_{\text {Helical }}\right)$ and for a fixed central flow rate $\left(V_{\text {Central }}\right)$.

\section{Summary}

It has been demonstrated experimentally that a permanent dynamo can be generated in a finite quasi-homogeneous domain of a well conducting fluid by a proper arrangement of spiral vortices within. The onset of self-exitation is characterized by a drastic increase of the pressure losses in the flow channels of the test module due to the effect of increasing Lorentz forces. As predicted by theory the mode of the observed magnetic field is non-axisymmetic. Two different magnetic states of opposite field direction were realized in the experiments, one by increasing the volumetric flow rates in a quasi-steady process, the other by an external magnetic pulse of opposite direction and adequate intensity.

\section{References}

Apel, A., Apstein, E., Rädler, K.-H., and Reinhardt, M.: Contribution to the theory of the planned Karlsruhe dynamo experiment, AIP-Report, 96, 1996.

Braginsky, S., Roberts, P. H.: Equations governing convection in the Earth's core and the geodynamo, Geophys. Astrophys. Fluid Dyn., 79, 1-97, 1995.

Busse, F. H.: Magnetohydrodynamics of the Earth's Dynamo, Annual Rev. Fluid Mech., 10, 435-462, 1978.

Busse, F. H. and Carrigan, C. R.: Laboratory Simulation of Thermal Convection in Rotating Planets and Stars, Science, 191, 81-83, 1976.

Busse, F. H.: Dynamo Theory of Planetary Magnetism and Laboratory Experiments, Springer Proceedings in Physics, 69, 197-208, Springer Verlag, 1992. 
Busse, F. H., Müller, U., Stieglitz, R., and Tilgner, A.: A two-scale homogeneous dynamo, an extended analytical model and an experimental demonstration under development, Magnetohydrodynamics, 32, 235-248, 1996.

Roberts, G. O.: Spatially periodic dynamos, Phil. Trans. R. Soc., London, A, 266, 535-558, 1970.

Roberts, G. O.: Dynamo action of fluid motions with twodimensional periodicity, Phil. Trans. R. Soc., London, A, 271, 411-454, 1972.

Stieglitz, R. and Müller, U.: Geodynamo - Eine Versuchsanlage zum Nachweis des homogenen Dynamoeffektes, FZKA Report FZKA-5716, 1996.

Stieglitz, R. and Müller, U.: Experimental demonstration of a homogeneous dynamo, Physics of Fluids, 13, 561-564, 2001.

Tilgner, A.: A kinematic dynamo with a small scale velocity field, Physics Letters A, 226, 75-79, 1997.

Tilgner, A.: Numerical simulation of the Karlsruhe dynamo; presented at the International Workshop on Homogeneous Dynamos, Forschungszentrum Karlsruhe 20-22 March 2000 and private communication, 2001. 